\title{
Antiproliferative agents and the prevention of scarring after surgery: friend or foe?
}

The use of antiproliferative agents to prevent scarring after surgery has revolutionised glaucoma filtration surgery in patients with a high risk of failure. The effectiveness of the postoperative 5-fluorouracil (5-FU) injection regimen developed by Parrish and the Miami group in the 1980s was proved conclusively in the multicentre fluorouracil filtering surgery study which increased the success rate in high risk eyes (previous failed filtration surgery or conjunctival incision cataract surgery) from $50 \%$ to $75 \%$. However, this regimen involved 21 injections of 5 -FU over 2 weeks, and even with lower dose regimens the injections are inconvenient and corneal erosions are common.

The use of antiproliferative agents has been accelerated by the advent of the regimen pioneered by Chen, involving only a single 5 minute intraoperative exposure to mitomycin $\mathrm{C}$ (MMC). This regimen has rapidly gained in popularity because of the ease of application, virtual absence of corneal side effects, and the improved long term intraocular pressure control in high risk eyes compared with the postoperative 5-FU injection regimen.

Inevitably, this seductively easy treatment has led to increasing suggestions that MMC should be used for all patients undergoing filtration surgery to achieve maximal pressure lowering. However, there are reasons why surgeons should tread with care until there has been further refinement of this treatment. Chronic hypotony following MMC use is the most commonly reported complication, and this may be associated with maculopathy and visual loss which is irreversible even after resolution of the hypotony. Many of the blebs are strikingly thin and totally avascular raising the spectre of a future harvest of persistent bleb leaks, hypotony, and endophthalmitis, particularly if bleb thinning increases as it often does even in blebs not treated with antiproliferative agents. The ocular complications after pterygium resection combined with local radiation treatment or topical MMC eyedrops (albeit with very much higher doses) such as scleromalacia, ulceration and ocular perforation, which only became apparent many years later, sound a note of caution for the future.

There is experimental evidence that MMC at the commonly used concentration of $0.4 \mathrm{mg} / \mathrm{ml}$ results in widespread cell death and virtually acellular scleral and conjunctival tissues for at least several months with no obvious recovery. Cellular repopulation by normal fibroblasts or vascular cells does not seem to occur, and what remaining cells there are in the tissue appear to be persistently abnormal in appearance and behaviour. Indeed, this is probably the reason MMC at higher concentrations works so well; the tissue is rendered incapable of mounting a healing response in the long term despite the persistent healing stimulus seen in conditions associated with a poor surgical prognosis such as neovascular glaucoma or chronic uveitis. It is vital that ophthalmologists appreciate the long term tissue effects they may be inducing with high concentration MMC and possibly other antiproliferative agents. It is also important for ophthalmologists to realise that the long term effects of these drugs are not restricted to the patient, and guidelines for the use of cytotoxic agents should be followed when handling these agents to protect themselves and their colleagues.

Attempts to reduce side effects while retaining the convenience of single application treatments include multiple tight but releasable sutures, anatomical valves, reductions in the concentration and time of exposure of $M M C$, or the use of different single application agents at doses which result in more reversible and less permanent effects on conjunctival and scleral cells for lower risk patients. In our hands the use of MMC (at currently used concentrations) is still restricted to high risk patients where the risk-benefit ratio clearly justifies its use, even taking into account possible long term complications. This may be particularly important in younger patients who have many years to develop long term complications.

It is very likely that antiproliferative agents will continue to be an increasingly important component of our therapeutic armamentarium in ophthalmology. As scarring is such a widespread problem in ophthalmology, antiproliferative agents including $\mathrm{MMC}$ are at present being tested in the laboratory and clinically in many situations including pterygium recurrence, proliferative vitreoretinopathy, post excimer laser scarring, optic nerve sheath fenestration, orbital and cutaneous scarring. Finally, there is the very exciting prospect that antiproliferative agents may provide us with the tools to determine whether or not long term lowering of the intraocular pressure to the low teens in glaucoma may arrest the disease process in the majority of patients. However, there is still a long way to go. It is important that we go forward using the extensive knowledge and experience in these areas, so that we tap the enormous potential of these agents, but in the safest way possible for our patients ensuring that we do not do more harm than good along the way.

Glaucoma Unit, Moorfields Eye Hospital

PENG T KHAW

and Wound Healing Group,

Department of Pathology,

Institute of Ophthalmology,

Bath Street, London EC1V 9EL 